\title{
Quality Evaluation of Carnitine for Proper Use of Supplement
}

\author{
Yuka Miyachi ${ }^{1}$, Chika Nakayama ${ }^{2}$, Taeyuki Oshima ${ }^{1}$ \\ ${ }^{1}$ College of Pharmacy, Kinjo Gakuin University, Nagoya, Japan \\ ${ }^{2}$ Faculty of Pharmacy Gifu University of Medical Science, Kani, Japan \\ Email: y-miyachi@kinjo-u.ac.jp, cnakayama@u-gifu-ms.ac.jp, t-oshima@kinjo-u.ac.jp
}

How to cite this paper: Miyachi, Y., Nakayama, C. and Oshima, T. (2021) Quality Evaluation of Carnitine for Proper Use of Supplement. Pharmacology \& Pharmacy, $12,55-62$.

https://doi.org/10.4236/pp.2021.122005

Received: January 15, 2021

Accepted: February 23, 2021

Published: February 26, 2021

Copyright $\odot 2021$ by author(s) and Scientific Research Publishing Inc. This work is licensed under the Creative Commons Attribution-NonCommercial International License (CC BY-NC 4.0). http://creativecommons.org/licenses/by-nc/4.0/ (c) (i) (2) Open Access

\begin{abstract}
In recent years, consumers are becoming more health-conscious. Supplements are becoming popular as they can be purchased easily. In Japan, the "Food with Function Claims" system began in 2015; the market for supplements is expected to continue to expand. However, the use of some supplements has not been supported with sufficient scientific evidence; some products have even caused health problems. In addition, consumers may not be able to make correct decisions based on the information from the Internet. Unlike medicine, the instruction on the usage of supplements is not precise. Therefore, improving the quality of the information on the supplements will become more necessary in the future. This study aims to improve the quality of the information on supplements by surveying the disintegration and dissolution behavior of the carnitine-containing supplements and evaluated their quality. The products tested here were supplements containing commercial carnitine. Disintegration test and dissolution test were conducted according to the Japanese Pharmacopoeia. Carnitine was quantified by high-performance liquid chromatography. The disintegration tests revealed that the products had different disintegration times, varying from 35 to 100 minutes; some products took more than 5 hours to disintegrate. Thus, some products had a slow rise in their dissolution rate. These results suggest that the carnitine-containing supplements used in this study may affect the absorption process. Therefore, in the case of oral administration, the expected effect might not be achieved depending on the product.
\end{abstract}

\section{Keywords}

Supplement, Disintegration Test, Dissolution Test, Carnitine 


\section{Introduction}

The concept of self-medication has permeated public consciousness in recent years. With the consumers' increasing health awareness, supplements have become an attractive option as they can be purchased and utilized easily by themselves. In 2015, "Foods with Function Claims" has been launched based on the Food Labeling Act. The system of "Foods with Function Claims" provides "opportunities for consumers to make voluntary and reasonable product choices." Supplements and functional foods are quickly forming a new food system. Food business operators are responsible for providing consumers with accurate information to help them understand the new system. By October 2020, about 3500 functional labeled foods have been reported, and the number of registered items is increasing [1]. In 2012, "Survey on the Use of Supplements" by the Consumer Committee of the Cabinet Office, about $25 \%$ of 10,000 consumers age 20 - 79 used supplements every day to "maintain health and promote health." If the occasional users are included, about $60 \%$ of the consumers have reported using supplements [2]. With the increasing prevalence of the Internet and smartphones, consumers have easy access to domestic and international products. The supplement market is expected to expand further in the future.

On the other hand, there have been cases of health hazards related to supplements. For example, 4 of 796 health claims against Chinese diet supplements were reported to be fatal [3]. In addition, much uncertain information from the Internet and television has significantly impacted food safety [4]. While the Internet allows instantaneous access to a vast amount of information, it is challenging for consumers to identify the correct information.

Moreover, supplements are different from medicines due to the lack of standardization in their quality control and usage [5]. First, supplements are sold by different companies. Also, the scientific evidence supporting their usage is not always sufficient. Even if the products have the same ingredients, they may significantly different compositions of the ingredients, resulting in variations in quality and safety. In addition, it has been reported that there are many cases of health claims caused by the use of products of deteriorated quality and overdose [6]. Kawamura et al. conducted disintegration tests on functional food products and found that they did not disintegrate within the specified time, indicating a clear difference in quality compared to medicines [7]. The National Consumer Affairs Center also conducted disintegration tests on popular health foods and found that in 42 out of 100 cases, the products did not disintegrate within the specified time [8]. Accurate information on supplements is necessary to the consumers to avoid the adverse events related to incorrect consumption. Therefore, there is a need for full disclosure of product quality information for medicine and supplements.

Carnitine, an ingredient of supplements, is thought to help control body weight and reduce body fat. Carnitine is a type of free amino acid found in living organisms and essential for lipid metabolism. It is touted as a "burning fat" etc., 
because it produces acyl-CoA from fatty acids in the body and uses it as energy [9] [10]. We have previously enhanced the quality of the information on supplements containing chondroitin sulfate, hyaluronic acid, and collagen by conducting disintegration and dissolution tests [11].

This study aims to improve the quality of the information on carnitine-containing supplements. The disintegration and dissolution behavior of the various carnitinecontaining supplements were evaluated based on the quality of the supplements.

\section{Method}

\subsection{Test Products}

The commercially available supplements (C1-C3) were selected for analysis. The ethical medication (M1) was used for comparison to the supplements. The content and additives of each supplement are shown (Table 1, Table 2). The reagents and solvents used in the experiment were reagent grade or high-performance liquid chromatography grade.

\subsection{Disintegration Test [12] [13]}

The disintegration test was conducted following the Japanese Pharmacopoeia 14th edition (JP14) and 16th edition (JP16). A bath of purified water at $37^{\circ} \mathrm{C} \pm 2{ }^{\circ} \mathrm{C}$ with the shaking speed of 29 - 32 shakes per minute and the shaking width of 53 - $57 \mathrm{~mm}$, and the disintegration tester HC-1 (Yazawa Science Co., Ltd.) were used. In JP14, auxiliary disks are always used (disk-method). In JP16, the use of auxiliary disks is permitted only where specified or allowed, and the disks are not used normally (no disk-method). The time from the start of the test to the tablet's disintegration was observed visually to determine the end of disintegration.

Table 1. Carnitine containing products.

\begin{tabular}{cccc}
\hline Type & Product name & Recommended dose & characteristics \\
\hline Supplement & C1 & 5 tablets/day $(750 \mathrm{mg})$ & Coating tablet \\
Medicine & C3 & 4 tablets/day $(432 \mathrm{mg})$ & Tablet \\
M1 & 6 tablets/day $(200 \mathrm{mg})$ & Coating tablet \\
\hline
\end{tabular}

Table 2. Additives agent.

C1

C2 piper longum powder, starch, reduced maltose, starch degradation product, green tea

C3 and vitamin $\mathrm{B}_{1}$

powdered reduced maltose, cellulose, calcium stearate, hydroxypropyl cellulose, shellac extract, cellulose, cyclodextrin, sucrose ester, fine silicon dioxide, hydrochloride, hydroxypropyl methylcellulose, shellac, glycerol, wax

cellulose, calcium stearate, starch (hydroxypropyl cellulose), tocotrienol, silicon dioxide, 


\subsection{Dissolution Test [12] [14]}

The dissolution test was conducted according to the method 2 (the paddle method), of the JP16. The dissolution tester TMB-81 NTR-1000 (Toyama Sangyo Co., Ltd.) was used. A bath of purified water at $37^{\circ} \mathrm{C} \pm 0.5^{\circ} \mathrm{C}$ with the paddle's rotational speed of $60 \mathrm{rpm}$ was used. The samples were eluted, collected, and passed through a $0.45-\mu \mathrm{m}$ membrane filter. Lastly, because some products did not increase the elution rate, an additional test using a stirrer at a rotation of about $100 \mathrm{rpm}$ was conducted instead of the paddle method (Figure 1).

\subsection{Quantification of Carnitine [12] [14]}

The amount of carnitine was measured using high-performance liquid chromatography (SHIMADZU CORPORATION) according to the JP16. The COSMOSIL5C 18 -MS-II $4.6 \times 150 \mathrm{~mm}$ column (NACALAITESQUE, INC.) was used at $40^{\circ} \mathrm{C}$ at a flow speed of $1.0 \mathrm{~mL} / \mathrm{min}$ and the wavelength $220 \mathrm{~nm}$. Acetonitrile/pH2.5 and $0.05 \mathrm{~mol} / \mathrm{L}$ phosphate buffer at the ratio of 1:19 were used for the mobile phase.

\section{Result}

\subsection{Disintegration Test}

The disintegration times of carnitine-containing products were determined (Table 3). JP stipulates that "tablets will disintegrate within 30 minutes, and coated tablets will disintegrate within 60 minutes." $\mathrm{C} 1$ was found to disintegrate in 52.4 minutes and C2 in 35.6 minutes using no disk-method. JP's stipulation concerns the regulation of clinical medicine. However, C1 and C2 were found to conform to the criterion. C3 had a sturdy film coating that did not break during the disintegration test; in fact, it did not disintegrate for more than 300 hours. Assayed by disk-method, all supplements were found to meet the standards of the JP. Assayed by the no disk-method, the supplements' disintegration times were found to be longer than that of the medicine.

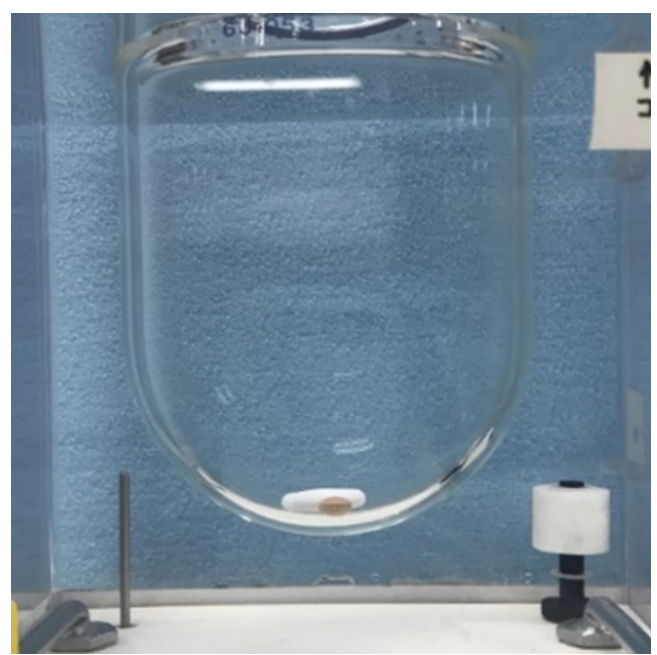

Figure 1. Dissolution test using stirrer. 


\subsection{Dissolution Test}

The dissolution of carnitine-containing products was assayed by the paddle method (Figure 2). C1 and C2 dissolved more slowly than M1. However, the dissolution rate in C2 and C3 after 120 minutes was about $100 \%$. On the other hand, C3 began to dissolve after 30 minutes, becoming 20\% dissolved after 120 minutes. The dissolution behavior of carnitine-containing products using the stirrer was also examined (Figure 3). When the stirrer was used instead of the paddle method, all the products dissolved more quickly. C3 was dissolved more slowly than $\mathrm{C} 1$ and $\mathrm{C} 2$; it became $100 \%$ dissolved 120 minutes.

Table 3. Disintegration time of carnitine ( $\mathrm{min})$.

\begin{tabular}{ccccc}
\hline & $\mathrm{C} 1$ & $\mathrm{C} 2$ & $\mathrm{C} 3$ & $\mathrm{M} 1$ \\
\hline no disk-method & $52.4 \pm 0.8$ & $35.6 \pm 1.4$ & $300<$ & 10.2 \\
disk-method & $32.8 \pm 0.7$ & $22.2 \pm 1.3$ & $42.1 \pm 10.2$ & $9.8 \pm 1.8$ \\
\hline
\end{tabular}

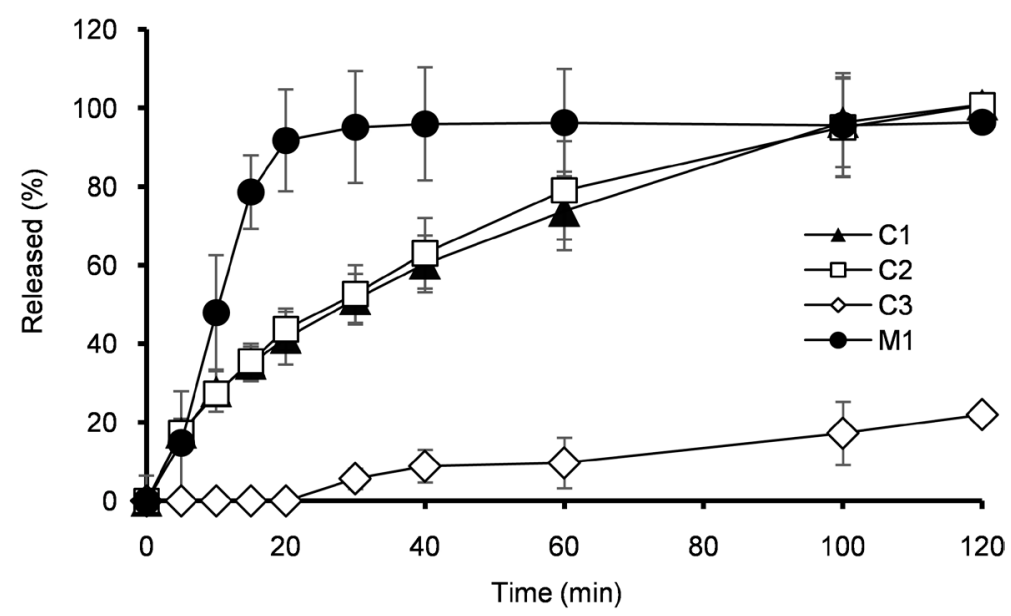

Figure 2. Dissolution behavior of paddle method. Mean \pm SD $(n=6)$.

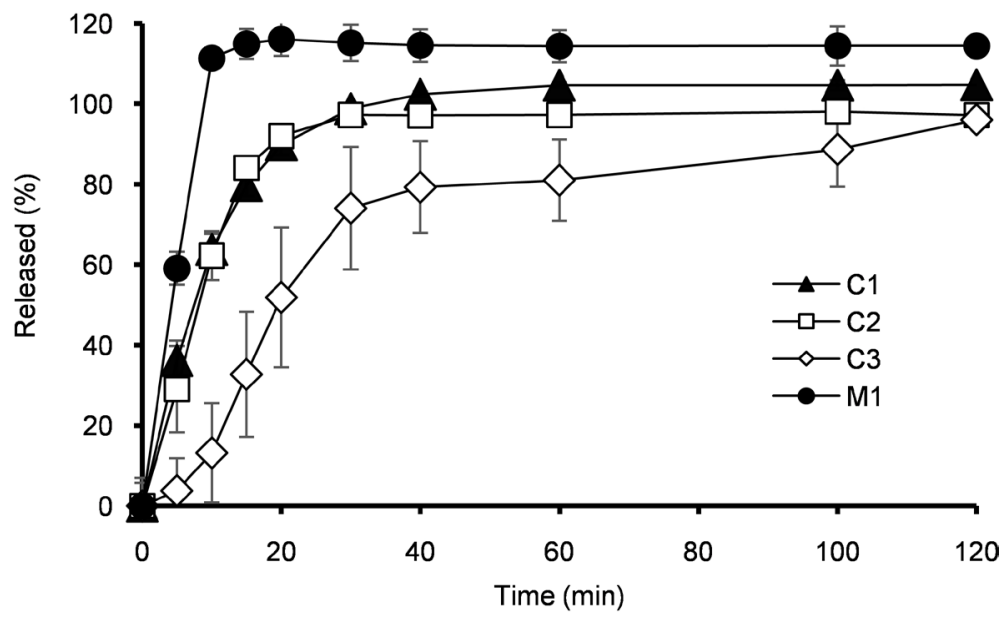

Figure 3. Dissolution behavior using stirrer. Mean \pm SD $(n=6)$. 


\section{Discussion}

As consumers become more health-conscious and increasingly promote self-medication, it becomes necessary and urgent to collect product information on dietary supplements. In this study, disintegration tests and dissolution tests were conducted on carnitine-containing products to increase the understanding of these supplements.

According to the results from the disintegration test of no disk-method, $\mathrm{C} 1$ and $\mathrm{C} 2$ were compliant with the regulation, and they displayed relatively small variability. C3 had a longer disintegration time and displayed higher variability, suggesting that its coating tablet was less likely to break under stimulation in the disintegration test. In reports on disintegration studies of functional foods, the percentage of coated tablet that did not disintegrate within the specified time was higher than that of uncoated tablet and capsules, which was similar to this study [15]. M1 had the shortest disintegration time and the smallest variability compared to the supplements.

According to the results from disk-method, all supplements became compliant with the regulation, and they all had shorter disintegration time. The C3 tablets became disintegrated by the method using disks. It was presumed that the disks facilitated the disintegration of C3's coating tablet, thus shortening the disintegration time. The disintegration time of $\mathrm{M} 1$ was not changed by the use of the disks. These results suggest that medicine can be effective regardless of the condition of the stomach contents.

When the paddle method was used for the dissolution test, C1 and C2 were shown to become $100 \%$ dissolved after 2 hours. Meanwhile, the dissolution behavior of $\mathrm{C} 3$ increased more slowly than that of $\mathrm{C} 1$ and $\mathrm{C} 2$, becoming $20 \%$ dissolved within the same time frame. The dissolution of $\mathrm{C} 3$ was likely affected by the nature of the film coating, as with its results from the disintegration test.

On the other hand, the increase in dissolution behavior was accelerated for all products using a stirrer. The dissolution rate of C3 increased to nearly $100 \%$. However, the rise in dissolution behavior was slower than that of the other products. C3's film coating might have taken some time to disintegrate; the physical stimulation by the stirrer might have accelerated the dissolution in all the products because of the fast start-up of dissolution.

According to the disintegration and dissolution tests, $\mathrm{C} 1 \mathrm{had}$ the shortest disintegration time and a dissolution rate of about $100 \%$. Among the supplements tested in this study, $\mathrm{C} 1$ is likely to achieve the fastest absorption and efficacy. C3 was considered to be difficult to achieve the expected effect according to the test method of the JP16. Considering the fact that they are absorbed after being disintegrated after ingested into the body, it can be inferred that they may be discharged from the body without being disintegrated, which may have some effect on the absorption process. The difference between the time of disintegration and the dissolution behavior seemed to have been caused by the difference in viscosity and collapsibility of the additives contained in the products. Moreover, such a 
difference may also affect the absorption process. In this study, some experiments were conducted with the additional stirrer, resulting in a reduction in the disintegration time and the increase of the dissolution rate. These data suggest that some products with film coating can have the expected effect with internal disintegration by the body, such as with or without food. In the case of insoluble coated tablets, it was not possible to evaluate according to the JP. Therefore, it is necessary to consider the body's stimulation and examine the experiment, such as physically destroying coating like this study.

The health support pharmacy system was started in April 2016. Health support pharmacies have the essential functions of the family pharmacist/pharmacy by proactively maintaining and promoting the health of community members [16]. Accordingly, pharmacists are required to be knowledgeable about pharmaceutical products as well as supplements [17]. Although the placebo effect may also be involved for supplements, it is thought that specific information needs to be provided so that formulation characteristics such as dissolution behavior and disintegration can be taken into account, just like for pharmaceuticals [18]. Therefore, it is necessary to present information on dosage and administration in consideration of formulation characteristics, such as dissolution behavior and disintegration. In the future, as a pharmacist, we must also educate consumers about supplements, recommend products that suit each consumer's needs, and contribute to the maintenance of health.

\section{Conflicts of Interest}

The authors declare no conflicts of interest regarding the publication of this paper.

\section{References}

[1] Consumer Affairs Agency, Government of Japan (2015) What Are Foods with Function Claims? https://www.caa.go.jp/policies/policy/food labeling/foods with function claims

[2] The Consumer Committee of the Cabinet Office (2012) Survey on the Use of Supplements.

https://www.cao.go.jp/consumer/iinkaikouhyou/2012/houkoku/201205 report.html

[3] Ministry of Health, Labor and Welfare (2006) In the Case of Chinese Diet Supplements. https://www.mhlw.go.jp/houdou/2002/07/h0719-3.html

[4] Umegaki, K. (2019) Safety and Effectiveness of Food with Health Claims and Its Effective Use. The Japanese Journal of Nutrition and Dietetics, 77, 67-75. https://doi.org/10.5264/eiyogakuzashi.77.67

[5] Sorin, S. (2020) Food Safety and Security, and for Rational and Autonomous Dietary Choices. Journal for the Integrated Study of Dietary Habits, 30, 159-166. https://doi.org/10.2740/jisdh.30.4 159

[6] Naito, H. (2013) Poisoning of Dietary Supplements and Chinese Herbs. Maruzen Publishing Co., Ltd., Tokyo.

[7] Kawamura, J., Nakamura, H., Muramatsu, A., Tajiri, Y. and Yamamoto, N. (2018) Disintegration Testing of Foods in the Form of Tablets and Capsules-Targeting 
Functional Foods. Journal of the Japan Pharmaceutical Association, 70, 19-23.

[8] National Consumer Affairs Center of Japan (2019) Quality Survey of Health Food Tablets and Capsules-They Might Look like Pharmaceutical Products, but Actually Not! http://www.kokusen.go.jp/e-hello/news/data/n-20190801 1.html

[9] Tajima, M. (2017) L-Carnitine-The Amounts in Foods and the Functional Properties. Bulletin of Jissen Women's University, Faculty of Human Life Sciences, 54, 1-5.

[10] Kondo, K. (2020) Evidence of Supplements to Improve Performance Directly. Journal of High Performance Sport, 5, 93-105.

[11] Miyachi, Y., Nakayama, C., Kato, A. and Oshima, T. (2018) Quality Evaluation of Glucosamine, Chondroitin Sulfate and Hyaluronic Acid for Proper Use of Supplement. Journal of the Japan Pharmaceutical Association, 70, 1475-1479.

[12] Japanese Pharmacopoeia 16th (2011) Disintegration, 116-117.

[13] Japanese Pharmacopoeia 14th (2001) Disintegration, 91-92.

[14] Pharmaceutical and Medical Device Regulatory Science Society of Japan, Japanese Orange Book, Levocarnitine Chloride.

[15] Kawamura, J., Muramatsu, A., Tajiri, Y. and Yamamoto, N. (2020) Disintegration Testing of Foods in the Form of Tablets and Capsules (Part 2)-Targeting Functional Foods. Journal of the Japan Pharmaceutical Association, 72, 17-22.

[16] Japan Pharmaceutical Association (2016) The Health Support Pharmacy System. https://www.nichiyaku.or.jp/kakaritsuke

[17] Chiba, T., Kobayashi, E., Nishijima, C. and Sato, Y. (2020) Consulting Pharmacists Who Have Advisory Staff License Regarding the Use of Medicines and Health Foods. Yakugaku Zasshi, 140, 723-728. https://doi.org/10.1248/yakushi.19-00256

[18] Nakano, S. (2013) Factors Contributing to the Placebo Effect. Japanese Pharmacology \& Therapeutics, 41, 723-728. 